Supporting Information for:

\title{
Conformational Landscape and Polymorphism in 5-Acetic Acid Hydantoin
}

\author{
B. A. Nogueira, ${ }^{1, *}$ G. O. Ildiz, ${ }^{1,2,{ }^{*}}$ J. Canotilho, ${ }^{3}$ M. E. S. Eusébio, ${ }^{1}$ \\ M. S. C. Henriques, ${ }^{4}$ J. A. Paixão ${ }^{4}$ and R. Fausto ${ }^{1}$ \\ ${ }^{1}$ CQC, Department of Chemistry, University of Coimbra, P-3004-535 Coimbra, Portugal. \\ 2 Faculty of Sciences and Letters, Department of Physics, Istanbul Kultur University, Atakoy Campus, \\ Bakirkoy 34156, Istanbul, Turkey. \\ ${ }^{3}$ Faculty of Pharmacy, University of Coimbra, Coimbra, Portugal. \\ ${ }^{4}$ CFisUC, Department of Physics, University of Coimbra, P-3004-516 Coimbra, Portugal.
}

\section{Index}

Figure S1. DSC heating curve from 25 to $222^{\circ} \mathrm{C}$ (heating rate $=10^{\circ} \mathrm{C} \mathrm{min}-1$ ) of polymorph I of $5 \mathrm{AAH}$.

Table S1.Definition of the internal coordinates used in the vibrational analysis performed on the most stable conformer of $5 \mathrm{AAH}$

Table S2. DFT(B3LYP)/6-311++G(d,p) optimized Cartesian coordinates for the different conformers of 5AAH. S4

Tables S3 to S11. Crystallographic tables for Polymorph I of 5AAH S9

* Corresponding authors’e-mails: ban@qui.uc.pt; g.ogruc@iku.edu.tr 


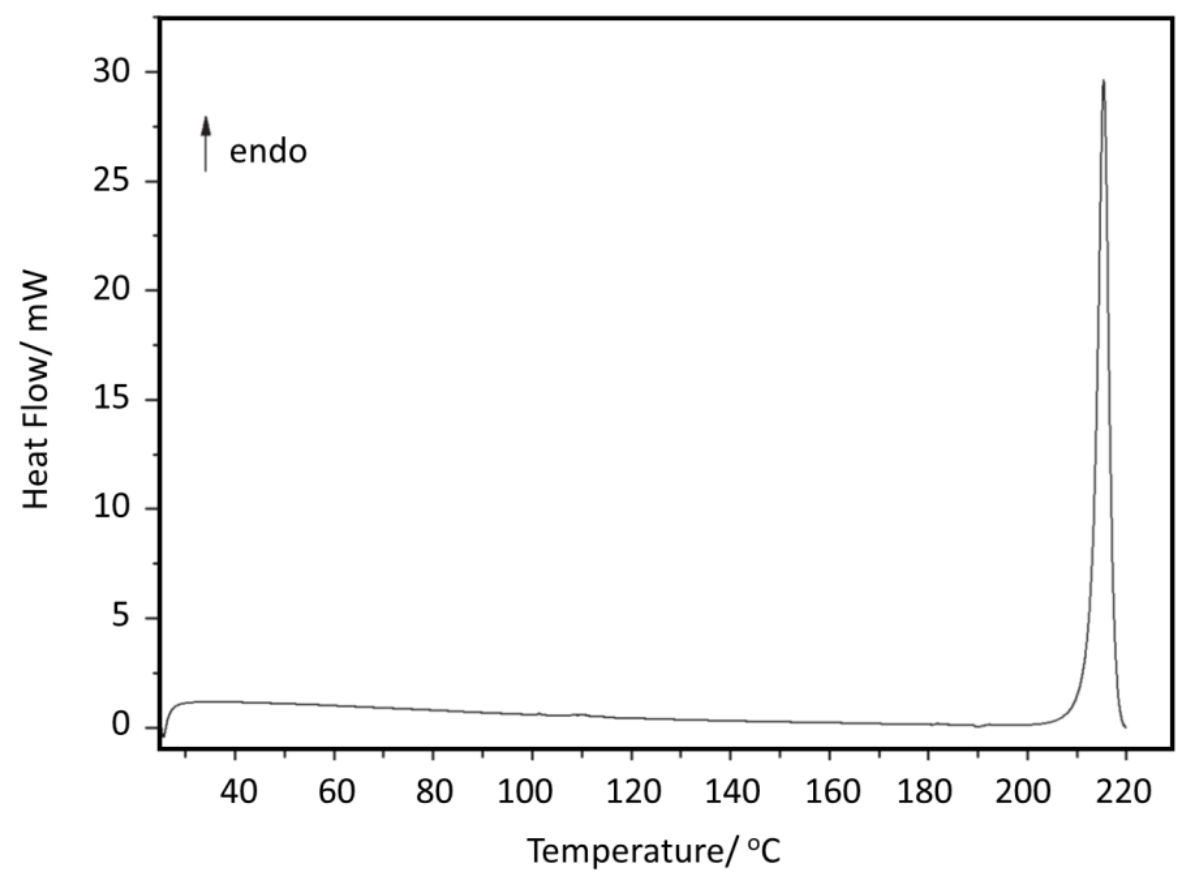

Figure S1. DSC heating curve from 25 to $222{ }^{\circ} \mathrm{C}$ (heating rate $=10^{\circ} \mathrm{C} \mathrm{min}-1$ ) of polymorph I of $5 A A H$ (mass $=1.61 \mathrm{mg}$ ) 
Table S1. Internal coordinates used in the normal modes analysis of 5AAH.

\begin{tabular}{|c|c|c|}
\hline Coordinate & Definition $a$ & $\begin{array}{l}\text { Approximate } \\
\text { description } b\end{array}$ \\
\hline $\mathrm{S}_{1}$ & $\mathrm{r} 1,6$ & $v\left(\mathrm{~N}_{1} \mathrm{H}\right)$ \\
\hline $\mathrm{S}_{2}$ & $\mathrm{r} 3,8$ & $v\left(\mathrm{~N}_{3} \mathrm{H}\right)$ \\
\hline $\mathrm{S}_{3}$ & $\mathrm{r}_{5,10}$ & $v(\mathrm{CH})$ \\
\hline $\mathrm{S}_{4}$ & $\mathrm{r}_{5,11}$ & $v\left(\mathrm{C}_{5} \mathrm{C}_{11}\right)$ \\
\hline $\mathrm{S}_{5}$ & $\mathrm{r} 2,7+\mathrm{r} 4,9$ & $v(\mathrm{C}=\mathrm{O})_{\mathrm{s}}$ \\
\hline S6 & $\mathrm{r} 2,7-\mathrm{r} 4,9$ & $v(\mathrm{C}=\mathrm{O})$ as \\
\hline $\mathrm{S}_{7}$ & $4 r_{3,4}-r_{5,4}-r_{1,5}-r_{2,1}-r_{3,2}$ & $v_{1}($ ring $)$ \\
\hline $\mathrm{S}_{8}$ & $\mathrm{r}_{5,4}+\mathrm{r}_{1,5}-\mathrm{r}_{2,1}-\mathrm{r}_{3,2}$ & $v_{2}($ ring $)$ \\
\hline $\mathrm{S}_{9}$ & $\mathrm{r}_{5,4}-\mathrm{r}_{1,5}-\mathrm{r}_{2,1}+\mathrm{r}_{3,2}$ & v3(ring) \\
\hline $\mathrm{S}_{10}$ & $\mathrm{r}_{5,4}-\mathrm{r}_{1,5}+\mathrm{r} 2,1-\mathrm{r}_{3,2}$ & $v_{4}($ ring $)$ \\
\hline $\mathrm{S}_{11}$ & $\mathrm{r}_{3,4}+\mathrm{r}_{5,4}+\mathrm{r}_{1,5}+\mathrm{r}_{2,1}+\mathrm{r}_{3,2}$ & v5(ring) \\
\hline $\mathrm{S}_{12}$ & $\mathrm{r}_{11,12}+\mathrm{r} 11,13$ & $v\left(\mathrm{CH}_{2}\right)_{\mathrm{s}}$ \\
\hline $\mathrm{S}_{13}$ & $\mathrm{r}_{11,12}-\mathrm{r}_{11,13}$ & $v\left(\mathrm{CH}_{2}\right)$ as \\
\hline $\mathrm{S}_{14}$ & $\beta_{2,4,3}-0.809 \beta_{3,5,4}-0.809 \beta_{3,1,2}+0.309 \beta_{1,4,5}+0.309 \beta_{2,5,1}$ & $\delta_{1}($ ring $)$ \\
\hline $\mathrm{S}_{15}$ & $-1.118 \beta_{3,5,4}+1.118 \beta_{3,1,2}-1.809 \beta_{1,4,5}+1.809 \beta_{2,5,1}$ & $\delta_{2}$ (ring) \\
\hline $\mathrm{S}_{16}$ & $\beta_{4,8,3}-\beta_{2,8,3}$ & $\delta\left(\mathrm{N}_{3} \mathrm{H}\right)$ \\
\hline $\mathrm{S}_{17}$ & $\beta_{3,7,2}-\beta_{1,7,2}$ & $\delta\left(\mathrm{C}_{2}=\mathrm{O}\right)$ \\
\hline $\mathrm{S}_{18}$ & $\beta_{2,6,1}-\beta 5,6,1$ & $\delta\left(\mathrm{N}_{1} \mathrm{H}\right)$ \\
\hline $\mathrm{S}_{19}$ & $\beta_{3,9,4}-\beta_{5,9,4}$ & $\delta\left(\mathrm{C}_{4}=\mathrm{O}\right)$ \\
\hline $\mathrm{S}_{20}$ & $-\beta_{1,11,5}-\beta_{4,11,5}-\beta_{1,10,5}-\beta_{4,10,5}+4 \beta_{11,10,5}$ & $\gamma(\mathrm{CH})$ \\
\hline $\mathrm{S}_{21}$ & $\beta_{1,10,5}-\beta_{4,10,5}$ & $\delta(\mathrm{CH})$ \\
\hline $\mathrm{S}_{22}$ & $\beta_{1,11,5}-\beta_{4,11,5}$ & $\delta\left(\mathrm{C}_{11}(\right.$ ring $\left.)\right)$ \\
\hline $\mathrm{S}_{23}$ & $\beta_{1,11,5}+\beta_{4,11,5}-\beta_{1,10,5}-\beta_{4,10,5}$ & $\gamma\left(\mathrm{C}_{11}\right.$ (ring) $)$ \\
\hline $\mathrm{S}_{24}$ & $4 \beta_{12,13,11}-\beta_{12,5,11}-\beta_{13,5,11}-\beta_{12,14,11}-\beta_{13,14,11}$ & $\delta\left(\mathrm{CH}_{2}\right)$ \\
\hline $\mathrm{S}_{25}$ & $\beta_{12,5,11}+\beta_{13,5,11}-\beta_{12,14,11}-\beta_{13,14,11}$ & $\mathrm{w}\left(\mathrm{CH}_{2}\right)$ \\
\hline $\mathrm{S}_{26}$ & $\beta_{12,5,11}-\beta_{13,5,11}-\beta_{12,14,11}+\beta_{13,14,11}$ & $\operatorname{tw}\left(\mathrm{CH}_{2}\right)$ \\
\hline $\mathrm{S}_{27}$ & $\beta_{12,5,11}-\beta_{13,5,11}+\beta_{12,14,11}-\beta_{13,14,11}$ & $\gamma\left(\mathrm{CH}_{2}\right)$ \\
\hline $\mathrm{S}_{28}$ & $\beta 5,14,11$ & $\delta(\mathrm{CCC})$ \\
\hline $\mathrm{S}_{29}$ & $\tau_{4,5,11,12}+\tau_{4,5,11,13}+\tau_{4,5,11,14}$ & $\tau\left(\mathrm{C}_{5}-\mathrm{C}_{11}\right)$ \\
\hline $\mathrm{S}_{30}$ & $\tau_{16,14,11,5}+\tau_{16,14,11,12}+\tau_{16,14,11,13}$ & $\tau\left(\mathrm{C}_{11}-\mathrm{C}_{14}\right)$ \\
\hline $\mathrm{S}_{31}$ & $0.309 \tau_{3,4,5,1}+0.309 \tau_{2,3,4,5}+0.809 \tau_{4,5,1,2}-0.809 \tau_{1,2,3,4}+\tau_{5,1,2,3}$ & $\tau_{1}($ ring $)$ \\
\hline $\mathrm{S}_{32}$ & $-1.118 \tau_{1,2,3,4}+1.118 \tau_{4,5,1,2}-1.809 \tau_{2,3,4,5}+1.809 \tau_{3,4,5,1}$ & $\tau_{2}($ ring $)$ \\
\hline $\mathrm{S}_{33}$ & $\gamma 6,5,1,2$ & $\gamma\left(\mathrm{N}_{1} \mathrm{H}\right)$ \\
\hline $\mathrm{S}_{34}$ & $\gamma 7,3,2,1$ & $\gamma\left(\mathrm{C}_{2}=\mathrm{O}\right)$ \\
\hline $\mathrm{S}_{35}$ & $\gamma 8,4,3,2$ & $\gamma\left(\mathrm{N}_{3} \mathrm{H}\right)$ \\
\hline $\mathrm{S}_{36}$ & $\gamma_{9,5,4,3}$ & $\gamma\left(\mathrm{C}_{4}=\mathrm{O}\right)$ \\
\hline $\mathrm{S}_{37}$ & $\mathrm{r} 14,15$ & $v\left(\mathrm{C}_{14}=\mathrm{O}\right)$ \\
\hline $\mathrm{S}_{38}$ & $\mathrm{r} 14,16$ & $v\left(\mathrm{C}_{14-\mathrm{O}}\right)$ \\
\hline $\mathrm{S}_{39}$ & $\mathrm{r} 16,17$ & $v(\mathrm{OH})$ \\
\hline $\mathrm{S}_{40}$ & $\mathrm{r}_{11,14}$ & $v\left(\mathrm{C}_{11}-\mathrm{C}_{14}\right)$ \\
\hline $\mathrm{S}_{41}$ & $\beta_{11,15,14}$ & $\delta(\mathrm{CC}=\mathrm{O})$ \\
\hline $\mathrm{S}_{42}$ & $\beta_{11,16,14}$ & $\delta(\mathrm{CCO})$ \\
\hline $\mathrm{S}_{43}$ & $\beta 14,17,16$ & $\delta(\mathrm{COH})$ \\
\hline $\mathrm{S}_{44}$ & $\tau_{15,14,16,17}$ & $\tau(\mathrm{OH})$ \\
\hline $\mathrm{S}_{45}$ & $\gamma_{11,15,14,16}$ & $\gamma\left(\mathrm{C}_{14}=\mathrm{O}\right)$ \\
\hline
\end{tabular}

a See Scheme 1 for atom numbering. $r$, bond distance; $\beta$, bond angle; $\gamma$, out-of-plane bending dihedral; $\tau$, torsional dihedral. $b v$, stretching; $\delta$, bending; $\gamma$, rocking; $\tau$, torsion; $w$, wagging; tw, twisting; $\mathrm{s}$, symmetric; as, anti-symmetric. 
Table S2. DFT(B3LYP)/6-311++G(d,p) optimized Cartesian coordinates $(\AA)$ for the different conformers of 5AAH.

$\begin{array}{lr}\text { cis-I } & \\ 7 & 0.606393000 \\ 6 & 1.947354000 \\ 7 & 2.285587000 \\ 6 & 1.236764000 \\ 6 & 0.055912000 \\ 1 & 0.164569000 \\ 8 & 2.708606000 \\ 1 & 3.211120000 \\ 8 & 1.229362000 \\ 1 & -0.160848000 \\ 6 & -1.192214000 \\ 1 & -1.003715000 \\ 1 & -1.463993000 \\ 6 & -2.361312000 \\ 8 & -2.322638000 \\ 8 & -3.480787000 \\ 1 & -4.186365000\end{array}$

$-0.999996000$

$-0.958776000$

0.415398000

1.241707000

0.305005000

$-1.860497000$

$-1.871960000$

0.736954000

2.448673000

0.380422000

0.682695000

0.615309000

1.721772000

$-0.214310000$

$-1.164921000$

0.165029000

$-0.454275000$

0.137217000

$-0.145358000$

$-0.165207000$

0.159871000

0.468395000

0.422766000

$-0.351076000$

$-0.408859000$

0.197298000

1.540441000

$-0.327032000$

$-1.403098000$

$-0.121347000$

0.004823000

0.748739000

$-0.644516000$

$-0.401720000$

cis-II

$\begin{array}{lr}7 & 0.589452000 \\ 6 & 1.936565000 \\ 7 & 2.278897000 \\ 6 & 1.225430000 \\ 6 & 0.031564000 \\ 1 & 0.143514000 \\ 8 & 2.697085000 \\ 1 & 3.210397000 \\ 8 & 1.221861000 \\ 1 & -0.206137000 \\ 6 & -1.190079000 \\ 1 & -0.970170000 \\ 1 & -1.421287000 \\ 6 & -2.456256000 \\ 8 & -3.519072000 \\ 8 & -2.273566000 \\ 1 & -3.128583000\end{array}$

$-1.000038000$

$-0.958498000$

0.413180000

1.237542000

0.300829000

$-1.867952000$

$-1.872761000$

0.736151000

2.443029000

0.364777000

0.709700000

0.631493000

1.760641000

$-0.083832000$

0.155289000

$-1.123171000$

$-1.570637000$

0.106534000

$-0.147800000$

$-0.137404000$

0.179309000

0.443906000

0.358611000

$-0.351531000$

$-0.355445000$

0.239457000

1.511979000

$-0.381404000$

$-1.450527000$

$-0.188502000$

$-0.135434000$

$-0.642040000$

0.724961000

0.821739000

\begin{tabular}{lrrr}
\multicolumn{4}{l}{ cis-III } \\
7 & 0.867723000 & -0.853148000 & 0.902254000 \\
6 & 1.871135000 & -0.745098000 & -0.032700000 \\
7 & 1.745273000 & 0.547116000 & -0.577956000 \\
6 & 0.713507000 & 1.273013000 & -0.033093000 \\
6 & 0.127573000 & 0.385301000 & 1.083686000 \\
1 & 0.910300000 & -1.593677000 & 1.584347000 \\
8 & 2.703157000 & -1.565599000 & -0.333894000 \\
1 & 2.288611000 & 0.846707000 & -1.374141000 \\
8 & 0.352351000 & 2.386286000 & -0.324436000 \\
1 & 0.382779000 & 0.866990000 & 2.036097000 \\
6 & -1.394931000 & 0.251778000 & 1.018089000 \\
1 & -1.837941000 & 1.248133000 & 1.122911000 \\
1 & -1.769364000 & -0.343911000 & 1.853904000 \\
6 & -1.903283000 & -0.334549000 & -0.281249000 \\
8 & -1.286828000 & -0.400933000 & -1.312479000 \\
8 & -3.179473000 & -0.766264000 & -0.154128000 \\
1 & -3.463011000 & -1.092617000 & -1.022102000
\end{tabular}


cis-IV

$\begin{array}{lrrr}7 & 0.679120000 & -0.877378000 & 0.888456000 \\ 6 & 1.722905000 & -0.879862000 & -0.006679000 \\ 7 & 1.771408000 & 0.425711000 & -0.534995000 \\ 6 & 0.824770000 & 1.268783000 & -0.002210000 \\ 6 & 0.088518000 & 0.440230000 & 1.069668000 \\ 1 & 0.581166000 & -1.641109000 & 1.538917000 \\ 8 & 2.457710000 & -1.791043000 & -0.299367000 \\ 1 & 2.405839000 & 0.680255000 & -1.277772000 \\ 8 & 0.622145000 & 2.424701000 & -0.277650000 \\ 1 & 0.365566000 & 0.873194000 & 2.039039000 \\ 6 & -1.442989000 & 0.491327000 & 0.954429000 \\ 1 & -1.732074000 & 1.540347000 & 0.830319000 \\ 1 & -1.907611000 & 0.119207000 & 1.867503000 \\ 6 & -2.020562000 & -0.309591000 & -0.194267000 \\ 8 & -2.921840000 & -1.101106000 & -0.095865000 \\ 8 & -1.416912000 & -0.021088000 & -1.369927000 \\ 1 & -1.831260000 & -0.567266000 & -2.055402000\end{array}$

cis-V

$\begin{array}{lll}7 & 1.366019000 & -1.140685000\end{array}$

$6 \quad 2.452559000 \quad-0.311019000$

$\begin{array}{lll}7 & 1.928000000 & 0.997031000\end{array}$

$\begin{array}{lll}6 & 0.566044000 & 1.043979000\end{array}$

$\begin{array}{lll}6 & 0.133581000 & -0.410649000\end{array}$

$\begin{array}{lll}1 & 1.526937000 & -2.085029000\end{array}$

$\begin{array}{lll}8 & 3.616089000 & -0.610885000\end{array}$

$\begin{array}{lll}1 & 2.502276000 & 1.803308000\end{array}$

$\begin{array}{lll}8 & -0.148941000 & 2.014604000\end{array}$

$1 \quad-0.168780000 \quad-0.495219000$

$6 \quad-1.045914000 \quad-0.824886000$

$1 \quad-1.106255000 \quad-1.918399000$

$1 \quad-0.913013000 \quad-0.487420000$

$6 \quad-2.367343000 \quad-0.333237000$

$8 \quad-2.642571000 \quad-0.281075000$

$8 \quad-3.233865000 \quad-0.006957000$

$1 \quad-4.058555000 \quad 0.277727000$

cis-VI

$\begin{array}{rrrr}7 & 1.390126000 & -1.129677000 & 0.167767000 \\ 6 & 2.437515000 & -0.267194000 & -0.055111000 \\ 7 & 1.859246000 & 1.017089000 & -0.121408000 \\ 6 & 0.503320000 & 1.020502000 & 0.116876000 \\ 6 & 0.127944000 & -0.443587000 & 0.413049000 \\ 1 & 1.588480000 & -2.066373000 & 0.483292000 \\ 8 & 3.609426000 & -0.524820000 & -0.179074000 \\ 1 & 2.399502000 & 1.839318000 & -0.348793000 \\ 8 & -0.245517000 & 1.965542000 & 0.092962000 \\ 1 & -0.171370000 & -0.507269000 & 1.464189000 \\ 6 & -1.016773000 & -0.946711000 & -0.481220000 \\ 1 & -1.132909000 & -2.024127000 & -0.319496000 \\ 1 & -0.784262000 & -0.794163000 & -1.535030000 \\ 6 & -2.365562000 & -0.313265000 & -0.203340000 \\ 8 & -3.147750000 & 0.038810000 & -1.044732000 \\ 8 & -2.625077000 & -0.245987000 & 1.125741000 \\ 1 & -3.492361000 & 0.173895000 & 1.230620000\end{array}$

0.097332000 $-0.061438000$ $-0.086265000$ 0.113049000 0.369363000 0.412760000 $-0.169331000$ $-0.285646000$ 0.076367000 1.419199000 $-0.520336000$ $-0.540887000$ $-1.548536000$ 0.036253000 1.208059000 $-0.944459000$ $-0.520780000$ 


\section{trans-I}

$\begin{array}{rrrr}7 & 0.617882000 & -1.003553000 & 0.117583000 \\ 6 & 1.960586000 & -0.937176000 & -0.155133000 \\ 7 & 2.276879000 & 0.443583000 & -0.155944000 \\ 6 & 1.212626000 & 1.247421000 & 0.170216000 \\ 6 & 0.049117000 & 0.286615000 & 0.468958000 \\ 1 & 0.188822000 & -1.873250000 & 0.395938000 \\ 8 & 2.737974000 & -1.834069000 & -0.367929000 \\ 1 & 3.197917000 & 0.782867000 & -0.392926000 \\ 8 & 1.178753000 & 2.454302000 & 0.212575000 \\ 1 & -0.170781000 & 0.343758000 & 1.541321000 \\ 6 & -1.207580000 & 0.656859000 & -0.317886000 \\ 1 & -1.009741000 & 0.594246000 & -1.394038000 \\ 1 & -1.471305000 & 1.696465000 & -0.095601000 \\ 6 & -2.371646000 & -0.261962000 & 0.021344000 \\ 8 & -2.289554000 & -1.206924000 & 0.757864000 \\ 8 & -3.550430000 & 0.037127000 & -0.574905000 \\ 1 & -3.470801000 & 0.821669000 & -1.131997000\end{array}$

\section{trans-II}

$7 \quad-0.495373000$

$6 \quad-1.862960000$

$7-2.250356000$

$6-1.232831000$

$6-0.002033000$

$1-0.104650000$

$8-2.584909000$

$1-3.195480000$

$8-1.283000000$

$1 \quad 0.243481000$

$6 \quad 1.206356000$

10.938483000

$1 \quad 1.456259000$

$6 \quad 2.422178000$

$8 \quad 3.293531000$

$8 \quad 2.451997000$

$1 \quad 1.676799000$

\section{trans-III}

$\begin{array}{lrrr}7 & 0.882621000 & -0.857868000 & 0.896095000 \\ 6 & 1.891028000 & -0.714179000 & -0.030396000 \\ 7 & 1.739158000 & 0.581606000 & -0.560917000 \\ 6 & 0.685184000 & 1.274222000 & -0.018029000 \\ 6 & 0.113632000 & 0.359287000 & 1.084685000 \\ 1 & 0.942960000 & -1.602602000 & 1.572208000 \\ 8 & 2.745802000 & -1.509931000 & -0.331328000 \\ 1 & 2.276090000 & 0.900316000 & -1.354142000 \\ 8 & 0.295209000 & 2.380890000 & -0.296840000 \\ 1 & 0.347908000 & 0.836737000 & 2.044753000 \\ 6 & -1.407177000 & 0.199159000 & 0.999231000 \\ 1 & -1.860570000 & 1.188830000 & 1.127930000 \\ 1 & -1.758940000 & -0.423564000 & 1.828901000 \\ 6 & -1.894947000 & -0.369082000 & -0.328915000 \\ 8 & -1.226131000 & -0.448732000 & -1.317982000 \\ 8 & -3.192266000 & -0.773646000 & -0.344300000 \\ 1 & -3.607120000 & -0.650979000 & 0.518254000\end{array}$

$$
\begin{array}{rr}
-0.948262000 & -0.297400000 \\
-0.988237000 & -0.073652000 \\
0.342359000 & 0.164157000 \\
1.253871000 & -0.018138000 \\
0.423424000 & -0.426411000 \\
-1.702444000 & -0.845032000 \\
-1.951910000 & -0.067861000 \\
0.589997000 & 0.420696000 \\
2.449045000 & 0.123253000 \\
0.666743000 & -1.466505000 \\
0.748070000 & 0.469946000 \\
0.575498000 & 1.517006000 \\
1.801137000 & 0.344514000 \\
-0.078840000 & 0.073032000 \\
0.313776000 & -0.649281000 \\
-1.350260000 & 0.557161000 \\
-1.524544000 & 1.107195000
\end{array}
$$




\section{trans-IV}

$7 \quad 0.888432000$

1.952204000

1.682375000

0.589254000

0.086981000

1.117600000

2.892875000

2.243322000

0.136088000

0.367007000

$-1.435019000$

$-1.913324000$

$-1.708603000$

$-2.080934000$

$-3.162295000$

$-1.396015000$

$-0.541788000$

$-0.812703000$

$-0.557743000$

0.687745000

1.330025000

0.402865000

$-1.415039000$

$-1.271063000$

1.068296000

2.399142000

0.882220000

0.198501000

1.171843000

$-0.395292000$

$-0.476607000$

$-0.157521000$

$-1.495660000$

$-1.618757000$

trans-V$$
7
$$

$$
1
$$

6

$1-1.110499000$

$1-0.920166000$

$6-2.392135000$

$8-2.651180000$

$8 \quad-3.305567000$

$\begin{array}{ll}1 & -2.920849000\end{array}$

\section{trans-VI}

$\begin{array}{lrrr}7 & -1.312596000 & -1.164970000 & -0.199883000 \\ 6 & -2.427668000 & -0.391542000 & -0.002878000 \\ 7 & -1.941403000 & 0.939747000 & 0.100718000 \\ 6 & -0.593064000 & 1.028460000 & -0.061002000 \\ 6 & -0.095629000 & -0.385706000 & -0.387259000 \\ 1 & -1.416479000 & -2.121622000 & -0.500979000 \\ 8 & -3.580976000 & -0.723621000 & 0.082675000 \\ 1 & -2.542197000 & 1.715729000 & 0.341799000 \\ 8 & 0.101304000 & 2.022042000 & 0.052283000 \\ 1 & 0.217465000 & -0.395903000 & -1.439529000 \\ 6 & 1.074817000 & -0.840631000 & 0.509041000 \\ 1 & 1.120202000 & -1.929286000 & 0.503686000 \\ 1 & 0.894260000 & -0.526157000 & 1.542454000 \\ 6 & 2.465189000 & -0.356185000 & 0.073956000 \\ 8 & 3.385037000 & -1.122803000 & -0.040365000 \\ 8 & 2.616902000 & 0.954953000 & -0.158550000 \\ 1 & 1.784726000 & 1.462862000 & -0.032757000\end{array}$

0.804734000 $-0.072107000$ $-0.647168000$ $-0.094636000$ 1.027546000 1.585354000 $-0.299005000$ $-1.396732000$ $-0.406014000$ 1.973399000 1.033347000 1.143544000 1.912909000 $-0.181225000$ $-0.587480000$ $-0.745775000$ $-0.292792000$

$-0.031027000$ $-0.070410000$ 0.030712000 0.190500000 0.302570000 0.208199000 $-0.180588000$ $-0.079633000$ 0.207855000 1.335280000 $-0.625121000$ $-0.794418000$ $-1.599251000$ 0.031726000 1.167890000 $-0.750717000$ $-1.599077000$ 


\section{trans-VII}

$7 \quad 0.960657000$

$6 \quad 1.938914000$

$7 \quad 1.674245000$

$6 \quad 0.569998000$

$6 \quad 0.126361000$

$1 \quad 1.010363000$

$8 \quad 2.839140000$

$1 \quad 2.168245000$

$8 \quad 0.014490000$

$1 \quad 0.403605000$

$6-1.391033000$

$1-1.875304000$

$1-1.621940000$

$6 \quad-1.976037000$

$8-2.359542000$

$8 \quad-2.048637000$

$1-1.702119000$

$\begin{array}{rr}-0.978951000 & 0.690700000 \\ -0.597688000 & -0.190442000 \\ 0.770552000 & -0.471124000 \\ 1.244357000 & 0.175475000 \\ 0.125769000 & 1.132673000 \\ -1.877716000 & 1.144252000 \\ -1.245782000 & -0.657268000 \\ 1.270500000 & -1.197182000 \\ 2.313073000 & 0.023495000 \\ 0.447803000 & 2.145718000 \\ -0.127690000 & 1.126928000 \\ 0.778763000 & 1.502636000 \\ -0.941333000 & 1.814819000 \\ -0.504346000 & -0.232655000 \\ -1.608930000 & -0.498762000 \\ 0.492773000 & -1.145512000 \\ 1.329294000 & -0.794777000\end{array}$


$5 \mathrm{AAH} \quad \mathrm{P} 212121 \mathrm{R}=0.03 \quad$ : Mar $2017: 16: 472020$

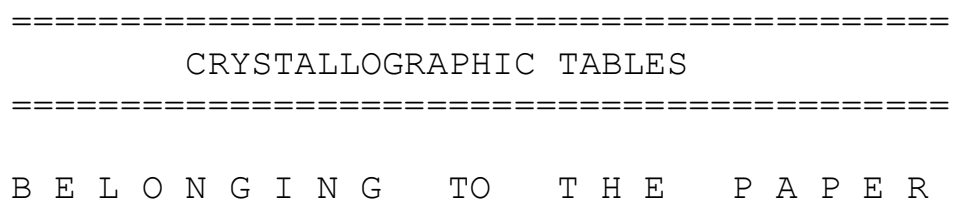

Conformational Landscape and Polymorphism in 5-Acetic Acid Hydantoin

b $y$

B. A. Nogueira, G. O. Ildiz, J. Canotilho, M. E. S. Eusébio,

M. S. C. Henriques, J. A. Paixão and R. Fausto

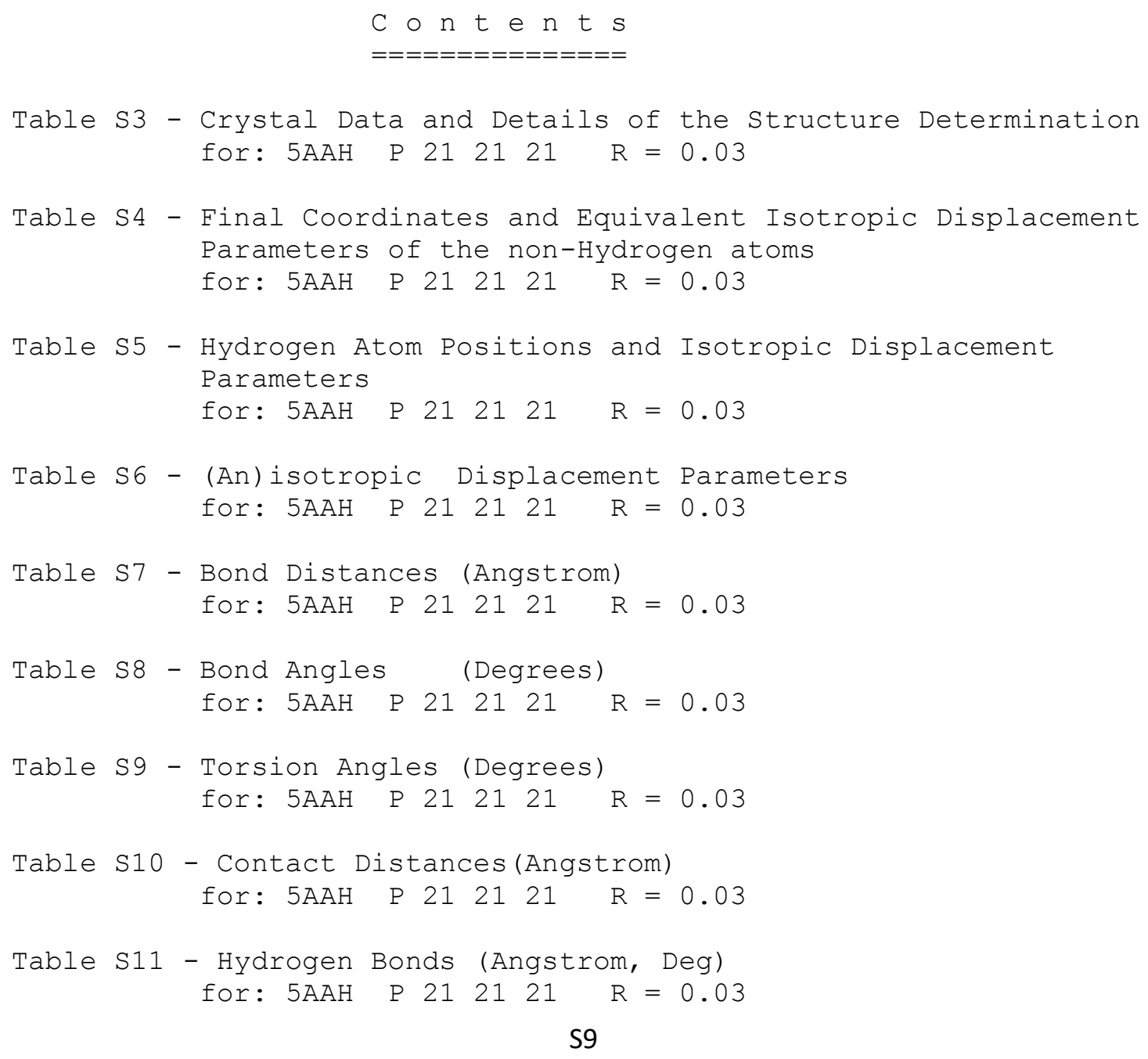




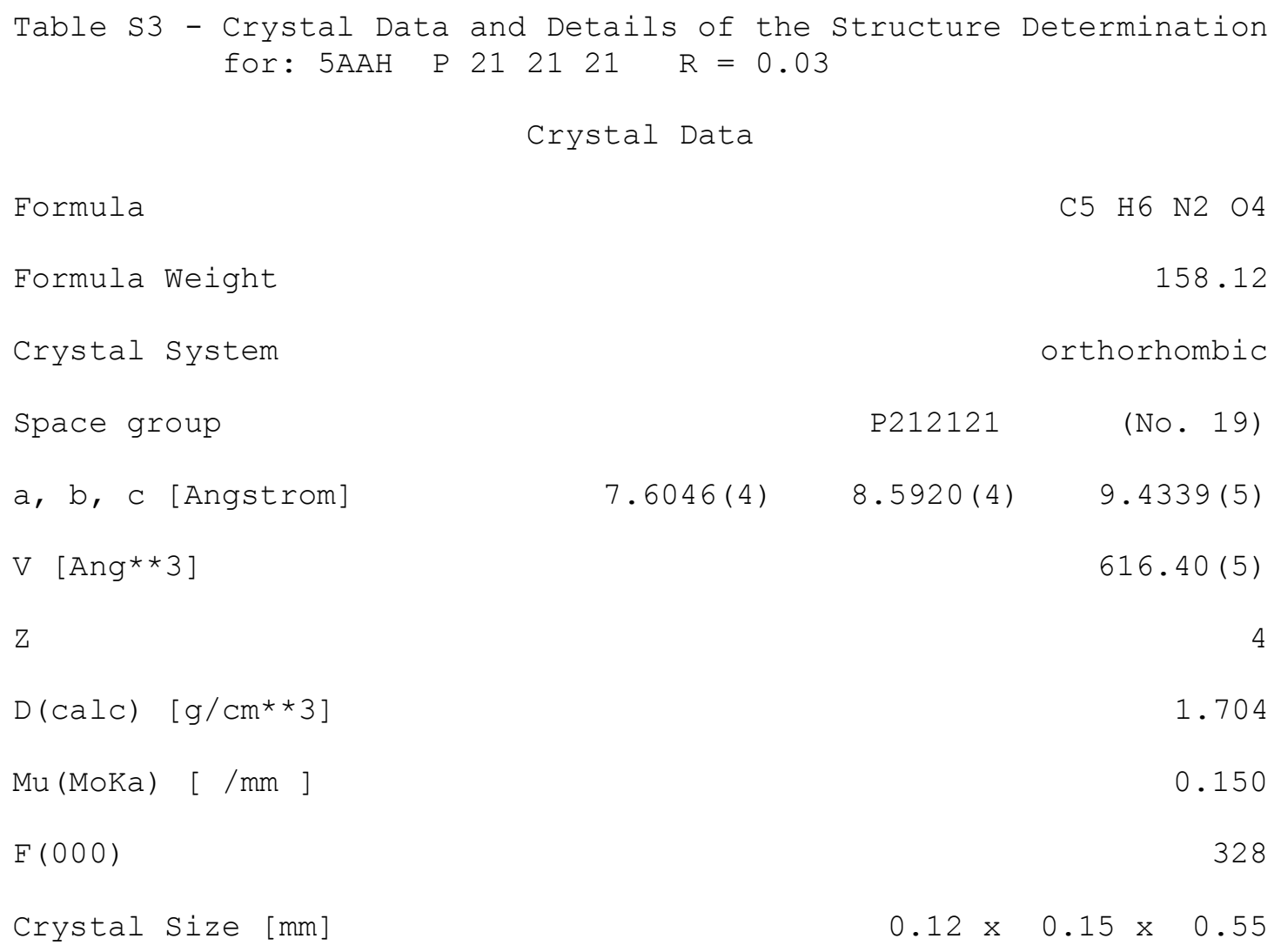

Formula

Formula Weight

Crystal system

Space group

$a, b, c$ [Angstrom $]$

$\mathrm{V}[$ Ang**3]

Z

$\mathrm{D}(\mathrm{calc}) \quad[\mathrm{g} / \mathrm{cm} * * 3]$

$\mathrm{Mu}(\mathrm{MoKa}) \quad[/ \mathrm{mm}]$

F $(000)$

Crystal Size [mm]

C5 $\begin{array}{llll}\mathrm{H} 6 & \mathrm{~N} 2 & \mathrm{O} 4\end{array}$

$$
158.12
$$

orthorhombic

P212121

(No. 19)

$7.6046(4)$

$8.5920(4)$

$9.4339(5)$

$616.40(5)$

1.704

0.150

328

$0.12 \times 0.15 \times 0.55$

Data Collection

Temperature (K)

Radiation [Angstrom]

MoKa

0.71073

Theta Min-Max [Deg]

$3.2, \quad 27.5$

Dataset

$-9: 9 ;-11: 11 ;-12: 12$

Tot., Uniq. Data, R(int)

$15800, \quad 1412, \quad 0.024$

Observed Data [I $>2.0$ sigma(I)]

1367

Refinement

Nref, Npar

1412, 112

$R$, wR2, $S$

$0.0252,0.0647,1.06$

$\left.\mathrm{W}={ }^{\wedge} 2^{\wedge}\left(\mathrm{FO} \mathrm{O}^{\wedge} 2^{\wedge}\right)+(0.0390 \mathrm{P}){ }^{\wedge} 2^{\wedge}+0.1014 \mathrm{P}\right]$ WHERE $\mathrm{P}=\left(\mathrm{FO} \mathrm{O}^{\wedge} 2^{\wedge}+2 \mathrm{FC} \mathrm{C}^{\wedge} 2^{\wedge}\right) / 3^{\prime}$

Max. and Av. Shift/Error

$0.00,0.00$

Flack $\mathrm{x}$

$0.8(13)$

Min. and Max. Resd. Dens. [e/Ang^3]

$-0.16,0.20$ 


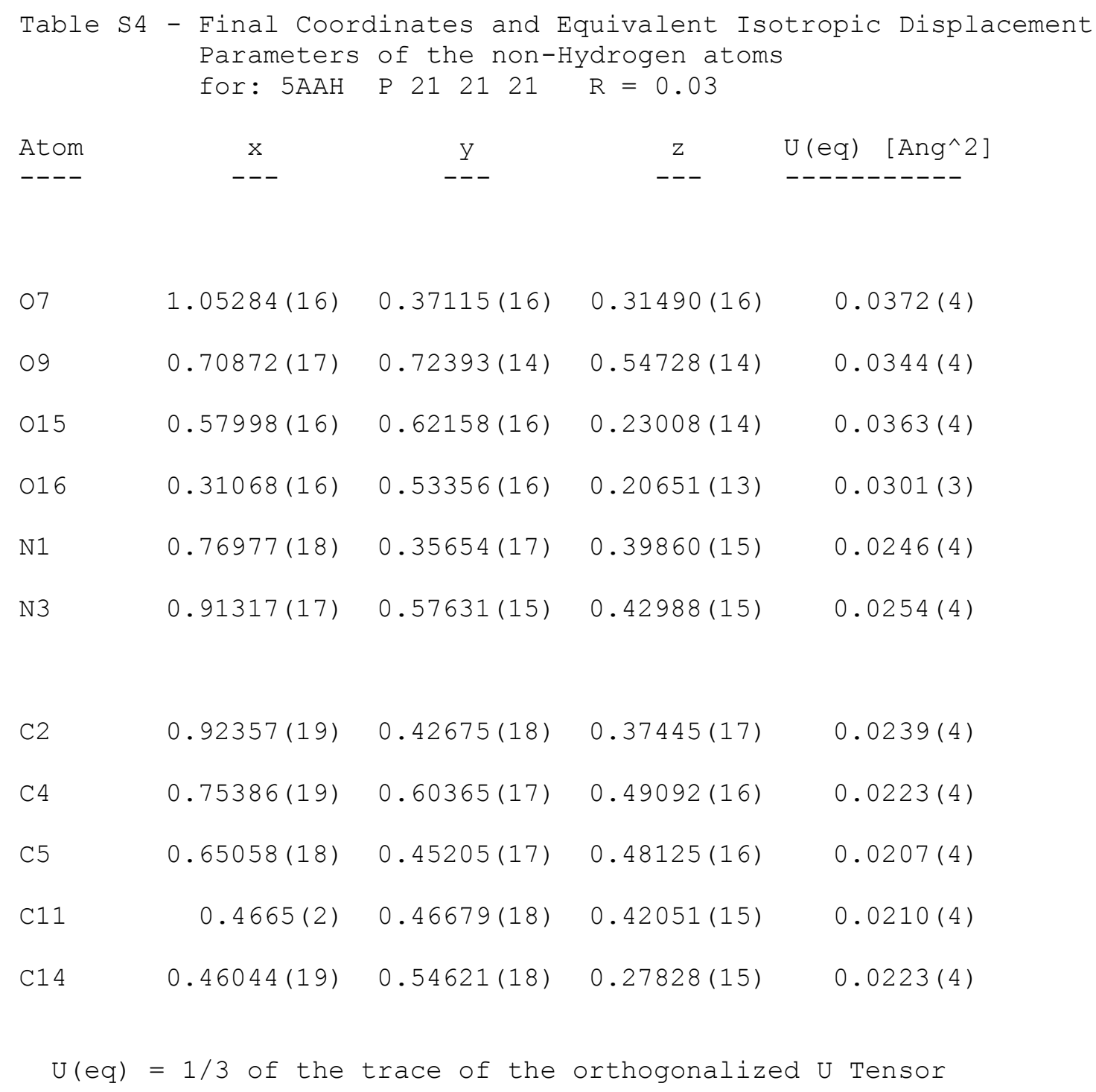




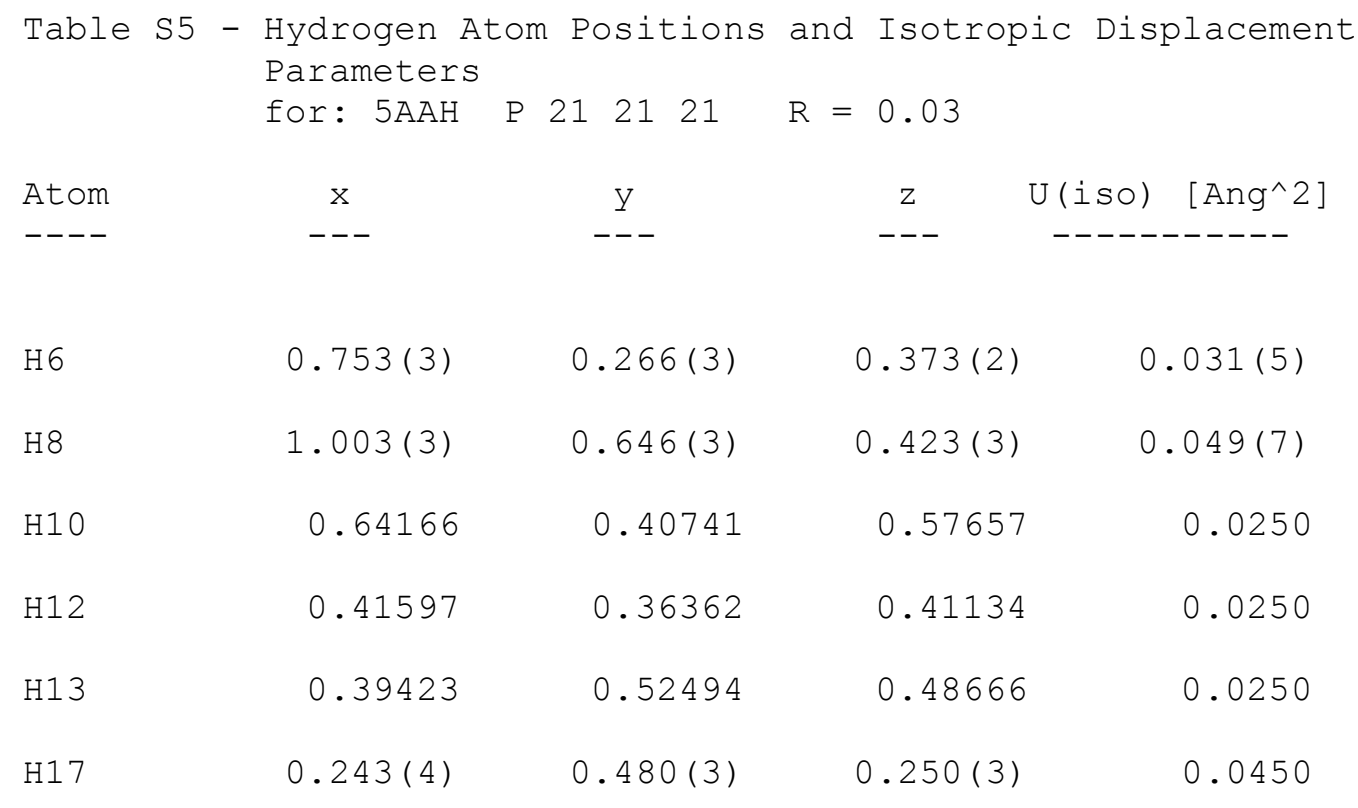

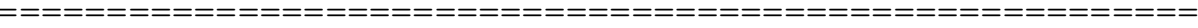

The Temperature Factor has the Form of Exp(-T) Where $\mathrm{T}=8 *(\mathrm{Pi} * 2) * \mathrm{U} *(\mathrm{Sin}(\mathrm{Theta}) /$ Lambda $) * * 2$ for Isotropic Atoms 


\begin{tabular}{|c|c|c|c|c|c|c|}
\hline Atom & $\mathrm{U}(1,1)$ or $\mathrm{U}$ & $\mathrm{U}(2,2)$ & $\mathrm{U}(3,3)$ & $\mathrm{U}(2,3)$ & $\mathrm{U}(1,3)$ & $\mathrm{U}(1,2)$ \\
\hline---- & ------ & ------ & ------ & ------ & ------ & ------ \\
\hline 07 & $0.0210(6)$ & $0.0335(7)$ & $0.0572(8)$ & $-0.0133(6)$ & $0.0052(6)$ & $0.0027(5)$ \\
\hline 09 & $0.0279(6)$ & $0.0265(6)$ & $0.0487(7)$ & $-0.0137(5)$ & $0.0006(6)$ & $0.0019(5)$ \\
\hline 015 & $0.0304(6)$ & $0.0426(7)$ & $0.0359(6)$ & $0.0155(5)$ & $0.0030(5)$ & $-0.0063(6)$ \\
\hline 016 & $0.0272(6)$ & $0.0347(6)$ & $0.0285(6)$ & $0.0047(5)$ & $-0.0073(5)$ & $-0.0006(5)$ \\
\hline N1 & $0.0206(7)$ & $0.0166(6)$ & $0.0366(7)$ & $-0.0041(5)$ & $-0.0018(5)$ & $0.0001(5)$ \\
\hline N3 & $0.0182(6)$ & $0.0199(6)$ & $0.0382(7)$ & $-0.0036(5)$ & $-0.0001(5)$ & $-0.0025(5)$ \\
\hline $\mathrm{C} 2$ & $0.0190(7)$ & $0.0213(7)$ & $0.0314(8)$ & $-0.0017(6)$ & $-0.0044(6)$ & $0.0013(6)$ \\
\hline $\mathrm{C} 4$ & $0.0197(7)$ & $0.0211(7)$ & $0.0261(7)$ & $-0.0012(6)$ & $-0.0031(6)$ & $0.0000(5)$ \\
\hline C5 & $0.0196(6)$ & $0.0200(6)$ & $0.0226(7)$ & $0.0016(5)$ & $-0.0011(5)$ & $-0.0010(6)$ \\
\hline $\mathrm{C} 11$ & $0.0170(6)$ & $0.0242(7)$ & $0.0217(7)$ & $0.0036(6)$ & $0.0011(5)$ & $-0.0023(6)$ \\
\hline C14 & $0.0215(7)$ & $0.0222(7)$ & $0.0232(7)$ & $0.0015(6)$ & $0.0013(6)$ & $0.0021(6)$ \\
\hline
\end{tabular}

The Temperature Factor has the Form of $\operatorname{Exp}(-\mathrm{T})$ Where $\mathrm{T}=8 *(\mathrm{Pi} * 2) * \mathrm{U} *(\mathrm{Sin}($ Theta) /Lambda)**2 for Isotropic Atoms $T=2 *(P i * 2) * \operatorname{Sumij}(h(i) * h(j) * U(i, j) * A s t a r(i) * A s t a r(j))$, for Anisotropic Atoms. Astar(i) are Reciprocal Axial Lengths and h(i) are the Reflection Indices. 


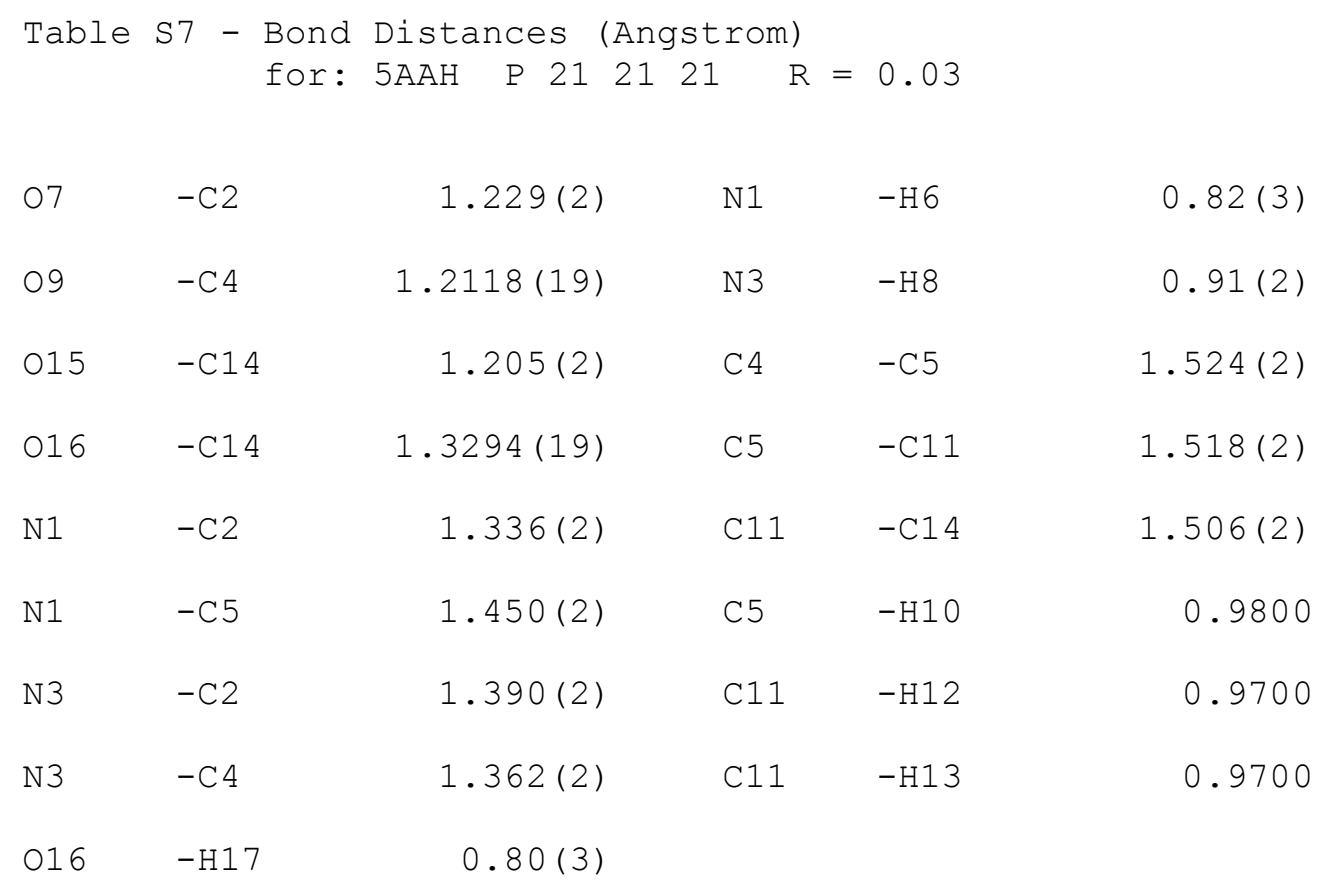




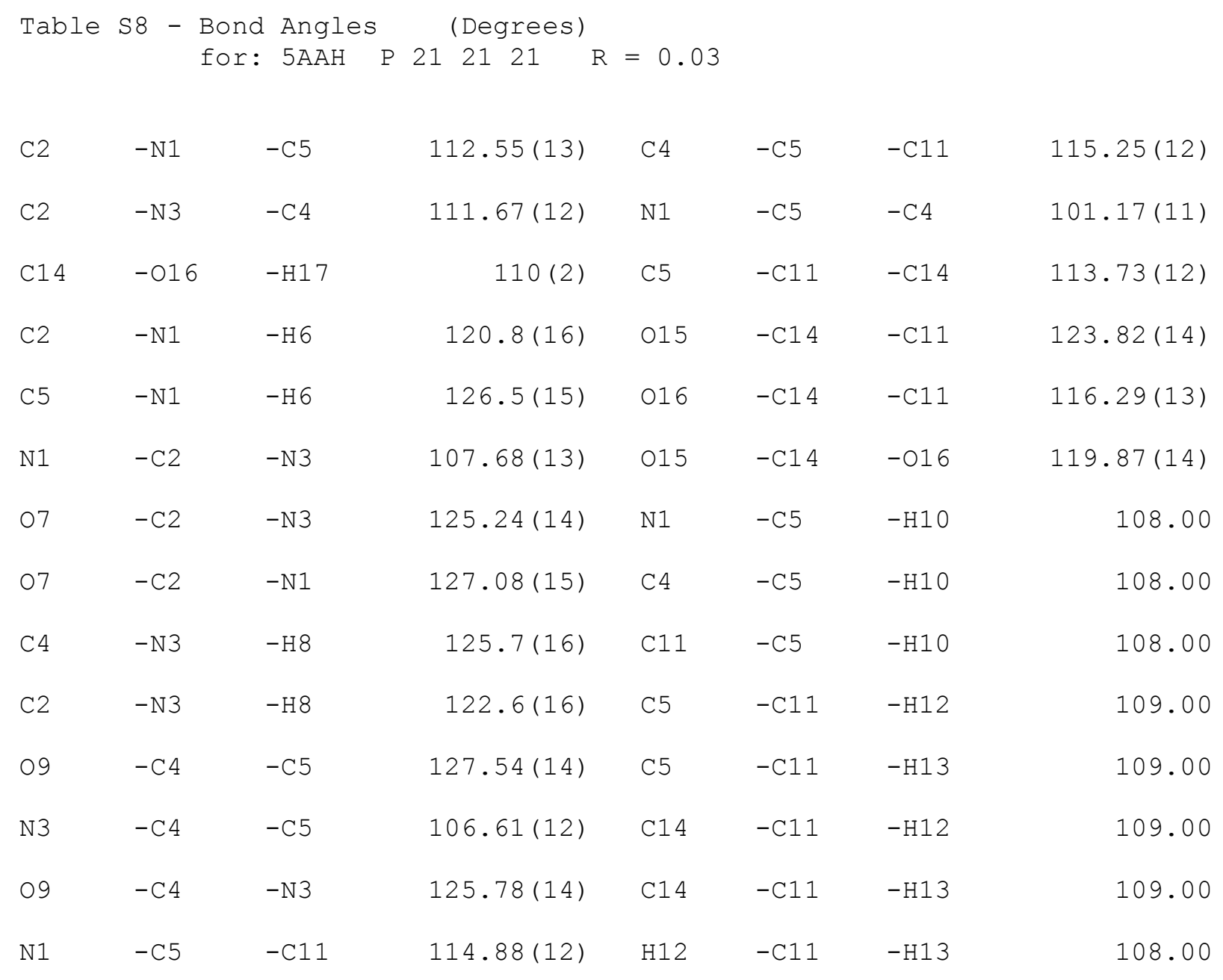




\begin{tabular}{|c|c|c|c|c|}
\hline & & : $5 \mathrm{AAH}$ & P 212 & $1 \quad R=0.03$ \\
\hline C5 & $-\mathrm{N} 1$ & $-\mathrm{C} 2$ & -07 & $175.53(16)$ \\
\hline C5 & $-\mathrm{N} 1$ & $-\mathrm{C} 2$ & $-\mathrm{N} 3$ & $-4.13(18)$ \\
\hline $\mathrm{C} 2$ & $-\mathrm{N} 1$ & $-\mathrm{C} 5$ & $-\mathrm{C} 4$ & $5.69(16)$ \\
\hline $\mathrm{C} 2$ & $-\mathrm{N} 1$ & $-\mathrm{C} 5$ & $-\mathrm{C} 11$ & $130.51(14)$ \\
\hline $\mathrm{C} 4$ & $-\mathrm{N} 3$ & $-\mathrm{C} 2$ & -07 & $-179.24(16)$ \\
\hline $\mathrm{C} 4$ & $-\mathrm{N} 3$ & $-\mathrm{C} 2$ & $-\mathrm{N} 1$ & $0.43(18)$ \\
\hline $\mathrm{C} 2$ & $-\mathrm{N} 3$ & $-\mathrm{C} 4$ & -09 & $-179.94(15)$ \\
\hline $\mathrm{C} 2$ & $-\mathrm{N} 3$ & $-\mathrm{C} 4$ & $-\mathrm{C} 5$ & $3.14(17)$ \\
\hline 09 & $-\mathrm{C} 4$ & $-\mathrm{C} 5$ & $-\mathrm{N} 1$ & $178.05(16)$ \\
\hline 09 & $-\mathrm{C} 4$ & $-\mathrm{C} 5$ & $-\mathrm{C} 11$ & $53.5(2)$ \\
\hline N3 & $-\mathrm{C} 4$ & $-\mathrm{C} 5$ & $-\mathrm{N} 1$ & $-5.11(15)$ \\
\hline N3 & $-\mathrm{C} 4$ & $-\mathrm{C} 5$ & $-\mathrm{C} 11$ & $-129.67(13)$ \\
\hline N1 & $-\mathrm{C} 5$ & $-\mathrm{C} 11$ & $-\mathrm{C} 14$ & $-63.33(17)$ \\
\hline C4 & $-\mathrm{C} 5$ & $-\mathrm{C} 11$ & $-\mathrm{C} 14$ & $53.73(17)$ \\
\hline $\mathrm{C} 5$ & $-\mathrm{C} 11$ & $-\mathrm{C} 14$ & -015 & $-15.7(2)$ \\
\hline $\mathrm{C} 5$ & $-\mathrm{C} 11$ & $-\mathrm{C} 14$ & -016 & $166.26(13)$ \\
\hline
\end{tabular}




\begin{tabular}{|c|c|c|c|c|c|}
\hline 07 & $.016 \_a$ & $2.6149(18)$ & 07 & . H17_a & $1.83(3)$ \\
\hline 07 & .C11_a & $3.401(2)$ & 07 & . H12_a & 2.9100 \\
\hline 09 & . C11_C & $3.316(2)$ & 07 &. $\mathrm{H} 6$ & $2.51(2)$ \\
\hline 09 & $.016 \_c$ & $3.2153(18)$ & 09 & . H13_C & 2.6000 \\
\hline 09 & . N3_d & $2.8361(18)$ & 09 & . H8_d & $1.94(2)$ \\
\hline 09 & .C14_C & $3.205(2)$ & 09 & . $\mathrm{H} 8$ & $2.61(2)$ \\
\hline 09 & . C11 & $3.115(2)$ & 09 & . $\mathrm{H} 10$ & 2.7800 \\
\hline 015 &. $\mathrm{~N} 3$ & $3.1819(18)$ & 015 & . $\mathrm{H} 1 \mathrm{O}_{-} \mathrm{e}$ & 2.5800 \\
\hline 015 & . C4_e & $3.230(2)$ & 015 &. $\mathrm{H} 17$ & $2.84(3)$ \\
\hline 015 & $.05 \_e$ & $3.1795(19)$ & 015 & . $\mathrm{H} 12$ f & 2.4700 \\
\hline 015 &. $\mathrm{C} 2$ & $3.389(2)$ & 016 & .H13_h & 2.6400 \\
\hline 015 &. $\mathrm{C} 4$ & $2.798(2)$ & 016 & . $H 6 \_f$ & $2.19(2)$ \\
\hline 015 &. $\mathrm{C} 5$ & $2.833(2)$ & 016 & . H13 & 2.7200 \\
\hline 015 &. $\mathrm{~N} 1$ & $3.130(2)$ & 016 &. $\mathrm{H} 12$ & 2.5500 \\
\hline 015 & . $c 2 \_e$ & $3.381(2)$ & N1 & . H12_b & 2.8300 \\
\hline 015 & . C11_f & $3.308(2)$ & N1 & . H12 & 2.6900 \\
\hline 016 & $.07 \_9$ & $2.6149(18)$ & $\mathrm{C} 2$ & .015 j & $3.381(2)$ \\
\hline 016 &.$N 1 \_f$ & $3.010(2)$ & $\mathrm{C} 2$ & .015 & $3.389(2)$ \\
\hline 016 & $.09 \_d$ & $3.2153(18)$ & C2 &. $\mathrm{C} 4$ & $2.277(2)$ \\
\hline N1 & . C14 & $3.079(2)$ & $\mathrm{C} 2$ & . C11 & $3.520(2)$ \\
\hline N1 & . C 4 & $2.298(2)$ & N3 & . H1O & 2.8800 \\
\hline N1 & $.016 \_i$ & $3.010(2)$ & C4 & $.015{ }^{j}$ & $3.230(2)$ \\
\hline N1 & .015 & $3.130(2)$ & C4 &. $\mathrm{N} 1$ & $2.298(2)$ \\
\hline N3 & .015 & $3.1819(18)$ & C4 & .015 & $2.798(2)$ \\
\hline N3 & $.09 \_C$ & $2.8361(18)$ & C4 & . C14 & $3.041(2)$ \\
\hline N3 &. $\mathrm{C} 5$ & $2.3157(19)$ & C5 &. N3 & $2.3157(19)$ \\
\hline
\end{tabular}




\begin{tabular}{|c|c|c|c|c|c|}
\hline 07 &. $\mathrm{H} 8$ & $2.60(3)$ & $\mathrm{C} 5$ & .015 & $2.833(2)$ \\
\hline 07 & .H10_b & 2.6900 & C5 & $.015 \_j$ & $3.1795(19)$ \\
\hline $\mathrm{C} 11$ &. $\mathrm{C} 2$ & $3.520(2)$ & $\mathrm{H} 10$ &. $\mathrm{C} 2$ & 2.8700 \\
\hline $\mathrm{C} 11$ & .09 & $3.115(2)$ & $\mathrm{H} 10$ &. $\mathrm{H} 6$ & 2.4300 \\
\hline $\mathrm{C} 11$ & $.09 \_d$ & $3.316(2)$ & $\mathrm{H} 10$ & .H12 & 2.3500 \\
\hline $\mathrm{C} 11$ & .07 _9 & $3.401(2)$ & $\mathrm{H} 10$ & . H13 & 2.3000 \\
\hline $\mathrm{C} 11$ & $.015_{i}$ & $3.308(2)$ & $\mathrm{H} 10$ & j.015_j & 2.5800 \\
\hline $\mathrm{C} 14$ & .09 d & $3.205(2)$ & $\mathrm{H} 10$ & $.07 \_k$ & 2.6900 \\
\hline $\mathrm{C} 14$ &. $\mathrm{~N} 1$ & $3.079(2)$ & $\mathrm{H} 12$ & $.07 \_9$ & 2.9100 \\
\hline $\mathrm{C} 14$ &. $\mathrm{C} 4$ & $3.041(2)$ & $\mathrm{H} 12$ & .016 & 2.5500 \\
\hline $\mathrm{C} 2$ & .H17_a & $2.74(3)$ & $\mathrm{H} 12$ & . N1 & 2.6900 \\
\hline $\mathrm{C} 2$ &. $\mathrm{H} 10$ & 2.8700 & $\mathrm{H} 12$ &. $\mathrm{H} 10$ & 2.3500 \\
\hline $\mathrm{C} 4$ & .H13 & 2.8200 & $\mathrm{H} 12$ & .H1 7 & 2.2500 \\
\hline $\mathrm{C} 4$ & . H $8 \_d$ & $2.99(2)$ & $\mathrm{H} 12$ & . N1_k & 2.8300 \\
\hline $\mathrm{C} 11$ &. $\mathrm{H} 17$ & $2.34(3)$ & $\mathrm{H} 12$ & .015 i & 2.4700 \\
\hline $\mathrm{C} 11$ &. $\mathrm{H} 6$ & $2.82(2)$ & $\mathrm{H} 13$ & .016 & 2.7200 \\
\hline $\mathrm{C} 14$ &.$H 6 \_f$ & $2.87(2)$ & $\mathrm{H} 13$ &. $\mathrm{C} 4$ & 2.8200 \\
\hline H6 & .07 & $2.51(2)$ & $\mathrm{H} 13$ &. $\mathrm{H} 1 \mathrm{O}$ & 2.3000 \\
\hline H6 &. $\mathrm{C} 11$ & $2.82(2)$ & $\mathrm{H} 13$ & .H17 & 2.5400 \\
\hline H6 & . H1O & 2.4300 & $\mathrm{H} 13$ & $.016 \_1$ & 2.6400 \\
\hline $\mathrm{H} 6$ & $.016 \_i$ & $2.19(3)$ & $\mathrm{H} 13$ & .09 d & 2.6000 \\
\hline H6 & .C14_i & $2.87(2)$ & $\mathrm{H} 17$ & $.07 \_9$ & $1.83(3)$ \\
\hline H8 & .07 & $2.60(3)$ & $\mathrm{H} 17$ & .015 & $2.84(3)$ \\
\hline H8 & .09 & $2.61(2)$ & $\mathrm{H} 17$ & . $22 \_9$ & $2.74(3)$ \\
\hline $\mathrm{H} 8$ & .09 _C & $1.94(2)$ & $\mathrm{H} 17$ &. $\mathrm{C} 11$ & $2.34(3)$ \\
\hline $\mathrm{H} 8$ & . C4_C & $2.99(2)$ & $\mathrm{H} 17$ & . H12 & 2.2500 \\
\hline $\mathrm{H} 10$ & .09 & 2.7800 & $\mathrm{H} 17$ &. $\mathrm{H} 13$ & 2.5400 \\
\hline $\mathrm{H} 10$ &. $\mathrm{~N} 3$ & 2.8800 & & & \\
\hline
\end{tabular}


Table S11 - Hydrogen Bonds (Angstrom, Deg)
for: 5AAH P $212121 \quad \mathrm{R}=0.03$

\begin{tabular}{|c|c|c|c|c|c|c|}
\hline $\mathrm{N} 1$ & $--\mathrm{H} 6$ & $\ldots 016$ & $0.82(3)$ & $2.19(3)$ & $3.010(2)$ & $175(2)$ \\
\hline N3 & $--\mathrm{H} 8$ & .. 09 & $0.91(2)$ & $1.94(2)$ & $2.8361(18)$ & $166(3)$ \\
\hline 016 & -- H17 & . 07 & $0.80(3)$ & $1.83(3)$ & $2.6149(18)$ & $166(3)$ \\
\hline C5 & -- H10 & .. 015 & 0.9800 & 2.5800 & $3.1795(19)$ & 120.00 \\
\hline C11 & -- H12 & .. 015 & 0.9700 & 2.4700 & $3.308(2)$ & 144.00 \\
\hline $\mathrm{C} 11$ & $--\mathrm{H} 13$ & .. 09 & 0.9700 & 2.6000 & $3.316(2)$ & 131.00 \\
\hline
\end{tabular}

